\section{Body Contouring - Intramuscular Gluteal Augmentation Following Massive Weight Loss: A Pilot Study}

\section{Abstract}

Background: Buttock-contouring surgery is becoming increasingly popular in plastic surgery practice. Nowadays, there are two main options to achieve gluteal augmentation: gluteal implants and fat grafting.

Aim and objectives: The purpose of this study was to evaluate the technique of gluteoplasty using a silicon implant and its mentioned complications in the literature in an attempt to eliminate the taboo surrounding this procedure as a step to implement it on a larger scale.

Materials and methods: A pilot study was performed to analyze the outcomes of gluteal augmentation in massive weight loss patient.

Results: The study showed that the gluteal augmentation could be done for those patients either alone or with other modality of treatment (fat grafting) with an acceptable rate of complications.

Keywords: Gluteal implant; Intramuscular gluteal augmentation; Body contouring surgery

Received: March 12, 2020; Accepted: April 15, 2020; Published: April 22, 2020
Rami Makkar, Mohammed Ashraf and Ahmed El-Naggar

Faculty of Medicine, Department of Surgery, Kasr Alainy Medical School, Cairo University, Manial, Cairo, Egypt

*Corresponding author: Ahmed El-Naggar

ફahmedrezk1000000@gmail.com

Lecturer of Plastic Surgery, Faculty of Medicine, Department of Surgery, Kasr Alainy Medical School, Cairo University, AlSaray Street, Manial, Cairo, Egypt.

Tel: +96560943060

Citation: Makkar R, Ashraf M, El-Naggar A (2020) Body Contouring - Intramuscular Gluteal Augmentation Following Massive Weight Loss: A Pilot Study. J Aesthet Reconstr Surg. Vol 6 No.1:1

\section{Introduction}

The form and size of the gluteal region have remained constant as symbols of maximum femininity. A beautiful "buttocks" are perceived as an important attribute of sexual attraction and beauty in every culture. Thus, improvement and cosmetic enhancement of the gluteal region has caused great interest among plastic surgeons [1,2].

Gluteal augmentation with silicone implants has been reported to yield complication rates as high as 38.1 percent [3], understandably why many surgeons are reluctant to attempt buttock augmentation with solid silicone implants or recommend it to their patients [4].

The paucity of data to guide treatment paradigms with no study of the overall complications or satisfaction rates is associated with the broad spectrum of techniques [5]. However, Gluteal augmentation with silicone implants may be the only remaining option to increase buttock volume, address ptosis and asymmetries in patients with great weight loss due to lack of donor tissue for liposculpture $[3,6]$.

This paper presents a pilot study of 5 patients who need the use of silicone implants in the buttocks after massive weight loss. The purpose of this study was to evaluate the technique and its mentioned complications in the literature attempting to eliminate the taboo surrounding this procedure as a step to implement it on larger scale.

\section{Materials and Methods}

A pilot study for 5 female patients suffered from Grade III gluteal ptosis [7] which is characterized by a skin fold angle of 30 or more; with or without extension of the lateral fold into the mid-portion of the thigh were selected for solid silicone gluteal augmentation. These patient with massive weight loss have lack in fatty tissue.

They have no health problems as active kidney or hepatic disease, diabetes mellitus, thyroid disease. Smokers, alcoholics, substance abusers and pregnant or lactating patients, were excluded from the study. Institutional verbal and written patient consents were obtained.

Measurements of the waist and the hip circumference (waist to hip ratio), in $\mathrm{cm}$ preoperatively and 3 months post surgery were used for assessment the patient in the standing position. 
The lateral fullness of the gluteal region, patient satisfaction and complications were documented.

The Intergluteal Incision and the intramuscular pocket dissection described by Vergara [8] and Gonzalez [9] were used for all patients. Four patients received biconvex gluteal implants $480 \mathrm{cc}$ and one patient received $530 \mathrm{cc}$ (Figures 1-3).

Following surgery they received the routine post operative medications and we stress upon good hygiene for the area of surgery to avoid infection of the wound and its sequelae. Patient were instructed never lie down on their back for 15 days and came back 1 week for first dressing.

\section{Results}

The age of patients ranged from 25 to 38 years old with a mean value 32.4 and the $\mathrm{BMI}$ of the patients ranged from 28 to 30 with a mean value 29 . All went through a smooth postoperative recovery and were discharged the following day post surgery.

Analysis of the waist to hip ratio (WHR) shows there was a significant difference in the "paired t test" between the pre and post WHR as the mean value was 0.83 and 0.78 respectively with " $\mathrm{t}$ " and " $\mathrm{p}$ " value of 8.85 and 0.0008 respectively.

The satisfaction rate of the patent was $60 \%$ where 2 patents were disappointed from the result. The contour of the gluteal region was corrected later by fat grafting and hayaluronic acid injections.

The lateral gluteal region correction failed in 3 patients (60\%) and 2 patients (66\%) complained regarding the hollowness of this region while 1 patient (33\%) was happy and appreciate the result.

Regarding the complications only 1 patient required re-suturing of the caudal part of the wound close to the anal verge by 2 interrupted sutures at the office under local anesthesia. No other major or minor complication were encountered (Tables 1 and 2) examples of the results are shown in Figures 4-10.

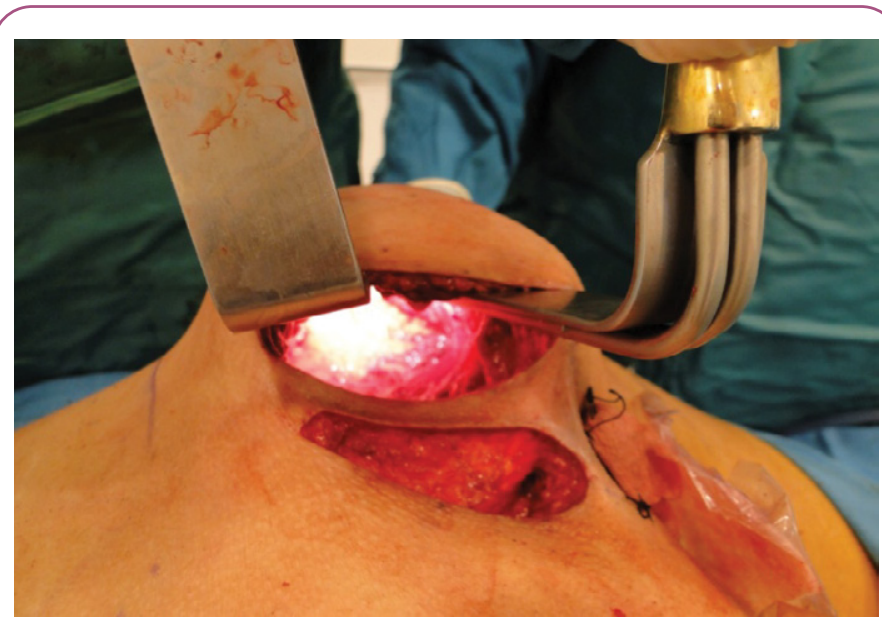

Figure 1

Intra operative picture showing an intramuscular pocket on the right side.

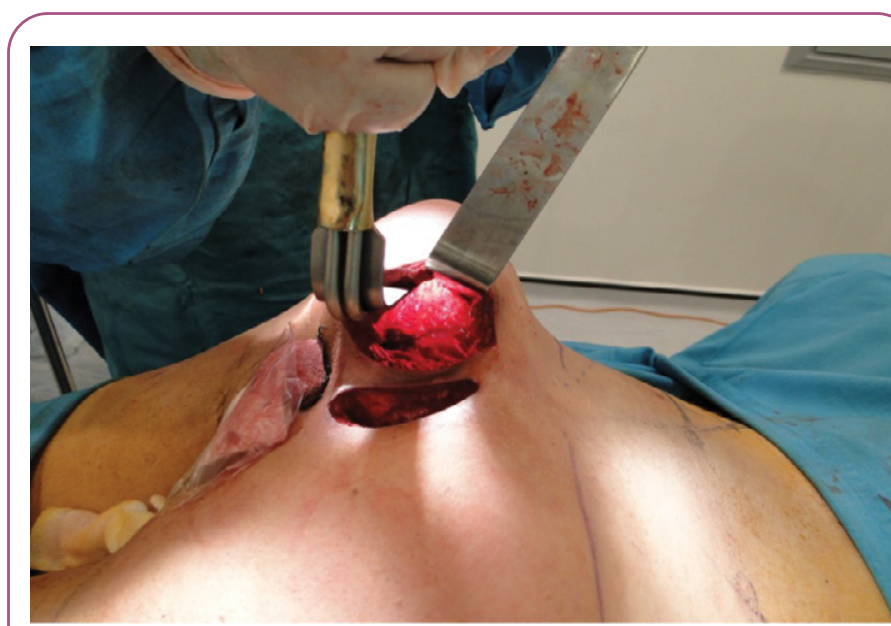

Figure 2 Intra-operative picture showing an intramuscular pocket on the left side.

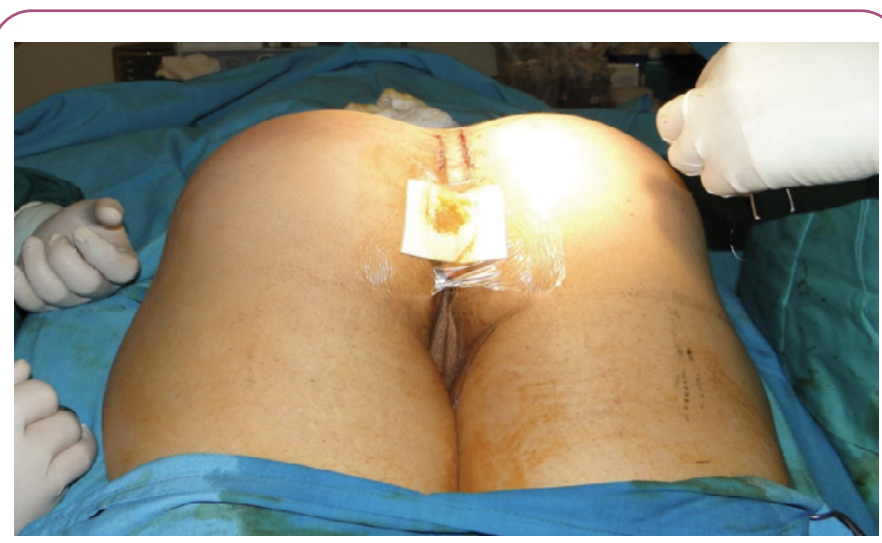

Figure 3 Intra-operative picture showing an immediate result after implant insertion.

Table 1 Patient characteristics showing Age, BMI, WHR and the lateral fullness.

\begin{tabular}{|c|c|c|c|c|c|c|c|c|c|}
\hline Patient & Age & BMI Waist & Hip & $\begin{array}{c}\text { WHR } \\
\text { before }\end{array}$ & Waist & Hip & $\begin{array}{c}\text { WHR } \\
\text { after }\end{array}$ & Lateral \\
fullness
\end{tabular}

\section{Discussion}

Universal aesthetic ideals of the female gluteal region, regardless of ethnicity, are defined by a ratio of the waist circumference at its narrowest to the thighs ("hips") circumference at the level of the maximum prominence of the buttocks (waist-to-hip ratio) equal to 0.7. Ethnic differences have been instead described by Roberts et al. as related to buttock size, lateral buttock fullness, and lateral thigh fullness [10]. 
Table 2 Result of the t-test showing the significance of the surgery.

\begin{tabular}{|c|c|c|}
\hline Alpha & \multicolumn{2}{|c|}{0.05} \\
\hline Hypothesized Mean Difference & \multicolumn{2}{|c|}{0} \\
\hline Mean & Variable 1 & Variable 2 \\
\hline & 0.830616482 & 0.7852985 \\
\hline Variance & Variable 1 & Variable 2 \\
\hline & 0.001944493 & 0.0011652 \\
\hline Observations & 5 & 5 \\
\hline Pearson Correlation & 0.9894531796 \\
\hline Observed Mean Difference & 0.045318002 \\
\hline Variance of the Differences & 0.0001309759 \\
\hline df & \multicolumn{2}{|c|}{4} \\
\hline $\mathrm{t}$ Stat & 8.8544173312 \\
\hline $\mathrm{P}(\mathrm{T}<=\mathrm{t})$ one-tail & 0.0004491854 \\
\hline $\mathrm{t}$ Critical one-tail & 2.1318467863 \\
\hline $\mathrm{P}(\mathrm{T}<=\mathrm{t})$ two-tail & 0.0008983708 \\
\hline $\mathrm{t}$ Critical two tail & 2.7764451052 \\
\hline
\end{tabular}

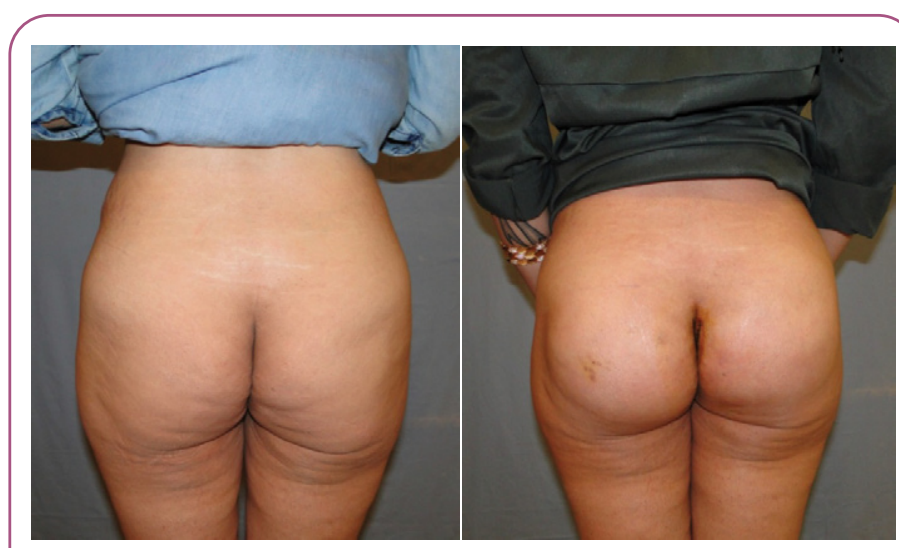

Figure 4 Case 1: Preoperative and postoperative back views.

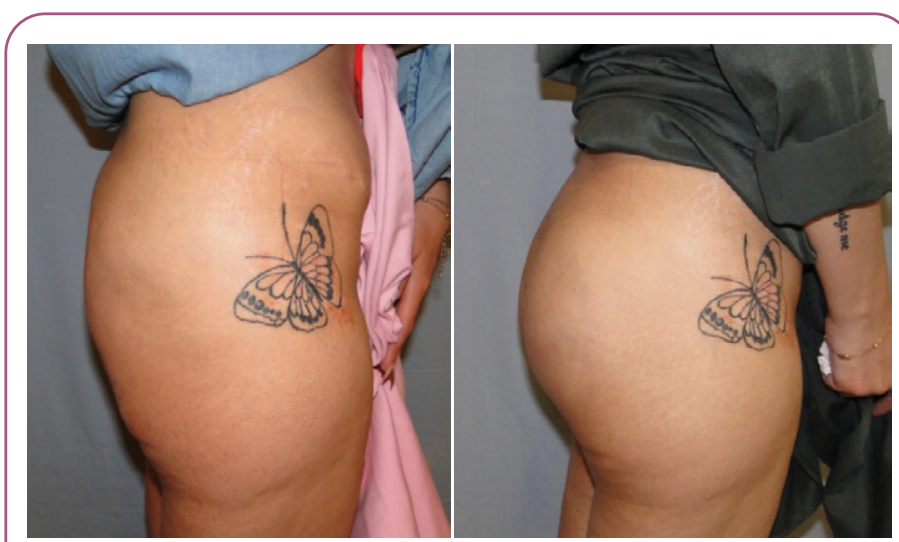

Figure 5 Case 1: Preoperative and postoperative lateral views.

Therefore, enhancement of the gluteal region became of great interest among plastic surgeons. Augmentation gluteoplasty may be performed through a number of different techniques, including biopolymer filler injection autologous fat grafting and silicone implant placement [3].

There is a broad range of biomaterials in the market which are

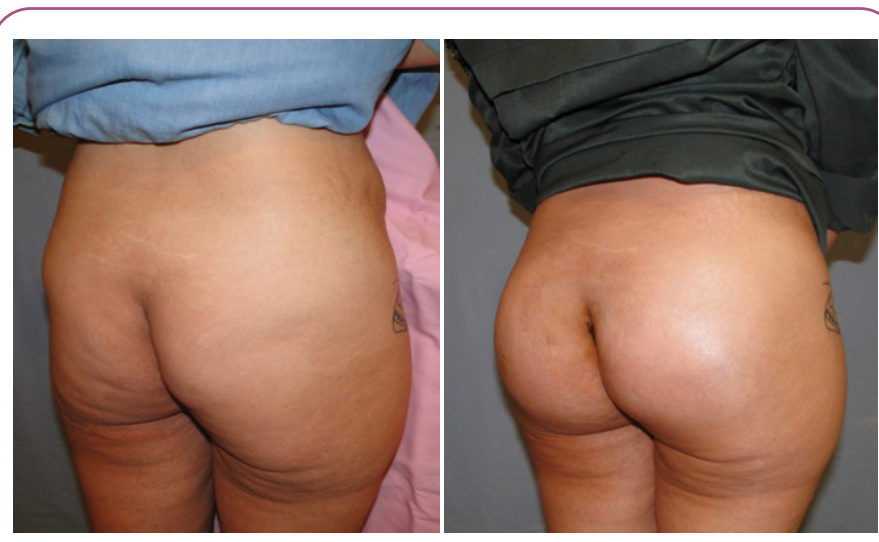

Figure 6 Case 1: Preoperative and postoperative lateral views.

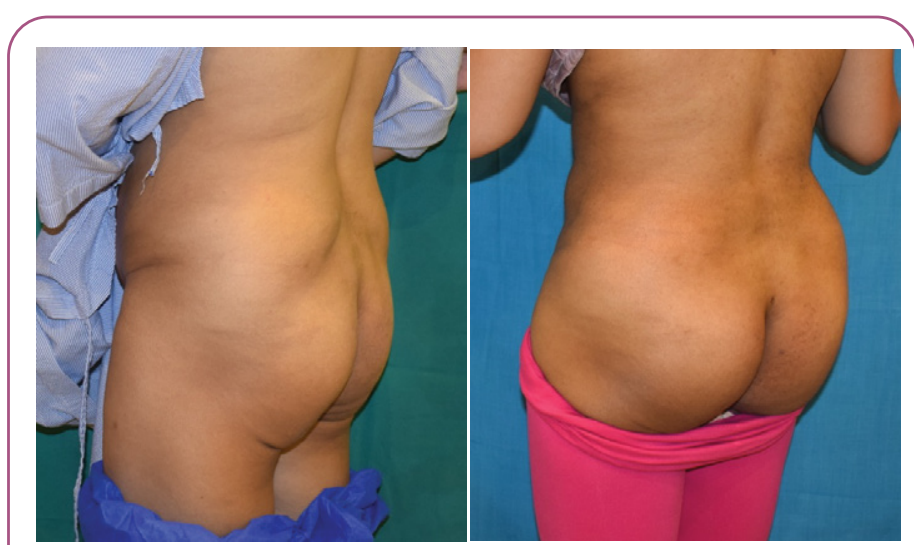

Figure 7 Case 2: Preoperative and postoperative left oblique views.

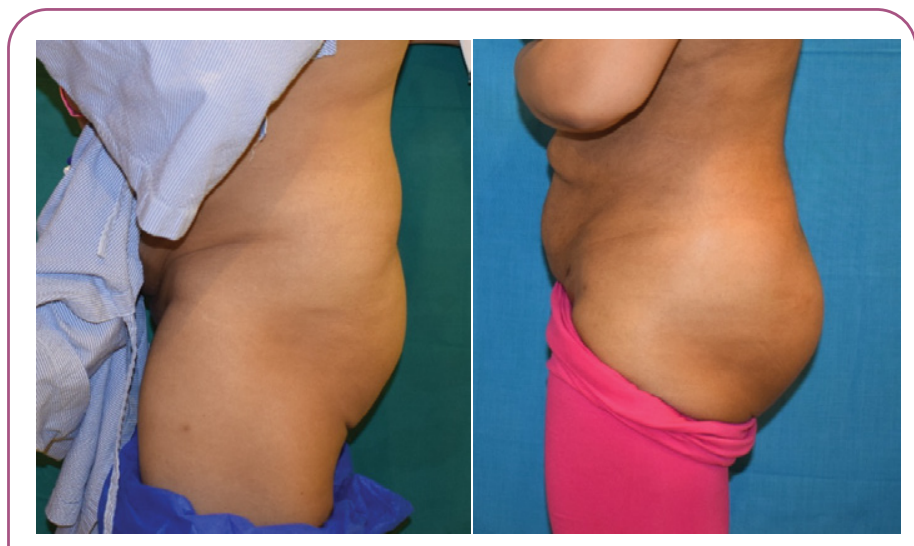

Figure 8 Case 2: Preoperative and postoperative left lateral views.

non authorized or even prohibited by the health authorities such as methacrylate, petrolate, mineral oils, polixane, hyaluronic acid. The tissue reaction produced by the inoculation of the mentioned materials is known as a foreign body granuloma or biopolymer granuloma.

The cells mainly involved in these lesions were predominantly macrophages, mast cells and fibroblasts, plasmatic cells, dendritic cells, and reactive lymphocytes. These materials cause early ( 1 hour post injection) and late (15 years post injection) 


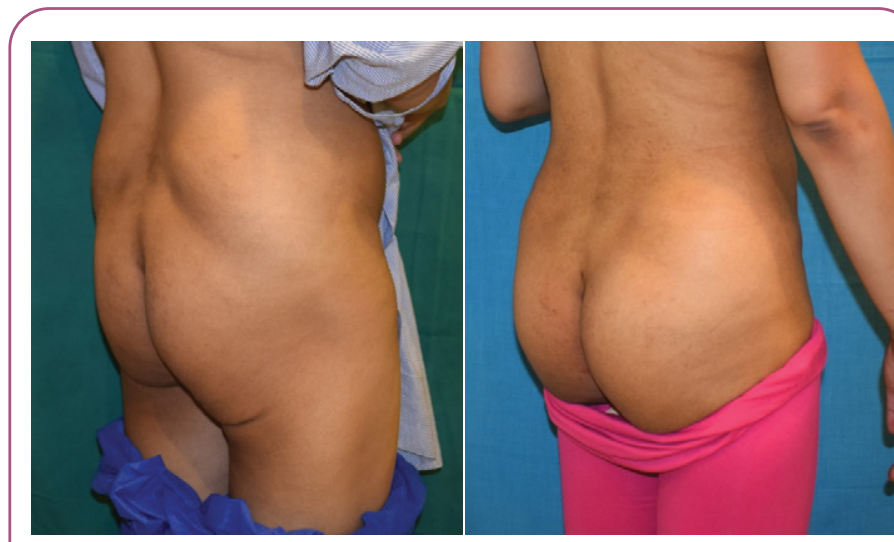

Figure 9 Case 2: Preoperative and postoperative right oblique views.

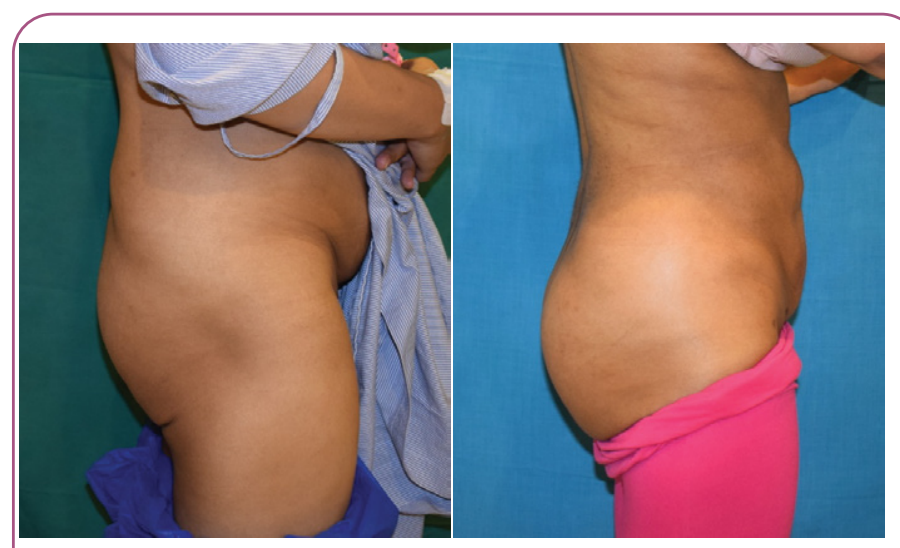

Figure 10 Case 2: Preoperative and postoperative right lateral views.

local and systemic symptoms. These symptoms include dermic manifestations such as simple skin hyperpigmentation but can cause autoimmune diseases such as Rheumatoid Arthritis, Lupus and Sjögren disease [11].

Fat grafting has an important role in gluteal augmentation and usually replace implant based gluteal augmentation making it occupy the third most infrequent procedure for body contouring [12]. For many patients specially following massive weight loss, gluteal augmentation with autologous fat grafting is not an option secondary to the lack of donor tissue. Fat transfer is not complications-free, and fatal risks associated with fat grafting, namely fat embolism may occur [13].

Gluteal dermolipectomy improves the skin excess and ptosis but worsens the projection due to flattening of the gluteal region. The autologous flaps have insufficient volume to improve the esthetics of the buttocks. They have the risk of steatonecrosis, denervation of the buttocks due to undermining, and insufficient to add volume in the lower pole of the gluteal region [13].

\section{References}

1 De la Pena JA, Rubio OV, Cano JP, Cedillo MC, Garces MT (2006) History of gluteal augmentation. Clin Plast Surg 33: 307-319.
Moreover, Scars can be disfiguring and aesthetically unpleasant and cause, anxiety, depression, and disruption of daily activities.

In spite of media suggestions to the contrary, scars cannot yet be made to disappear [14]. Throughout its history, the techniques have been improved by refining placement of the implant while changing the location and type of incision used [15].

Buttocks implant surgery has evolved greatly since the introduction of the intramuscular technique described by Vergara and Marcos in 1996 [8]. This technique has provided the achievement of more natural, safe, and long-lasting results compared to other gluteoplasty augmentation techniques.

Implants may be placed in any one of four anatomical planes relative to the gluteus maximus muscle: subcutaneous, subfascial, submuscular, or intramuscular [12]. However the intramuscular position provides the greatest amount of implant coverage and pads the implant with muscle tissue above and below as well as along the inferior edge [7].

Gluteal augmentation surgery suffers from a reputation of high rates of complications among internationally recognized high qualified surgeons reaching up to (38.1\%) [6] while in other survey the complication rate was lower than that $30.5 \%$ [5] or even 21.6 percent [3]. The most common postoperative complications are seroma, dehiscence, ptosis, capsular contracture, noticeable implants, infection and the need for implant removal. The intramuscular technique has a lower complication rates.

Gluteal augmentation with implants is less indicated than autologous fat grafting as it doesn't reach the ideal waist-to-hip ratio of 0.7 and does not allow for the reshaping of the lateral third of the buttocks and is considered highly controversial. The use of high projection implant and minimal fat grafting to the lateral third may solve the problem and could be the only choice in massive weight loss patients. In our study, implant solved the problem statistical but the final hourglass appearance anticipated by most of the patients was not achieved.

\section{Conclusion}

Buttock augmentation with solid silicone implants is a safe procedure with acceptable complications. The intramuscular position and meticulous surgical technique reduce the complication rate. Gluteal augmentation with intramuscular silicone implant can be an option to treat patients with gluteal lipoatrophy following massive weight loss. The result achieved is natural and could be more enhanced through adjunct procedures.

\section{Ethical Approval}

This study reviewed and approved by Ethics Committee at the Faculty of Medicine, Cairo University, Egypt. 
3 Sinno S, Chang JB, Brownstone ND, Saadeh PB, Wall S (2016) Determining the safety and efficacy of gluteal augmentation: a systematic review of outcomes and complications. Plast Reconstr Surg 137: 1151-1156.

4 Senderoff DM (2011) Buttock augmentation with solid silicone implants. Aesthetic Surg J 31: 320-327.

5 Oranges CM, Tremp M, Di Summa PG, Haug M, Kalbermatten DF, et al. (2017) Gluteal augmentation techniques: a comprehensive literature review. Aesthetic Surg J 37: 560-569.

6 Mofid MM, Gonzalez R, De la Peña JA, Mendieta CG, Senderoff DM, et al. (2013) Buttock augmentation with silicone implants: a multicenter survey review of 2226 patients. Plast Reconstr Surg 131: 897-901.

7 Mendieta CG (2006) Classification System for Gluteal Evaluation. Clin Plast Surg 33: 333-346.

8 Vergara R, Marcos M (1996) Intramuscular gluteal implants. Aesthetic Plast Surg 20: 259-262.

9 Gonzalez R (2010) Gluteal implants: The "XYZ" intramuscular method. Aesthetic Surg J 30: 256-264.
10 Roberts TL, Weinfeld AB, Bruner TW, Nguyen K (2006) "Universal” and ethnic ideals of beautiful buttocks are best obtained by autologous micro fat grafting and liposuction. Clin Plast Surg 33: 371-394.

11 Gutierrez-Gonzalez LA, De Zohbi BP, Perez-Alfonzo R (2012) Psoriatic Arthritis post biopolymer injection. Revista Latinoamericana de Psoriasis y Artritis Psoriásica 6: 41-48.

12 Flores-Lima G, Eppley BL, Dimas JR, Navarro DE (2013) Surgical pocket location for gluteal implants: a systematic review. Aesthetic Plast Surg 37: 240-245.

13 Levan P, Habre BS (2017) Gluteal implants versus autologous flaps in patients with post-bariatric surgery weight loss: A prospective comparative study of 3-dimensional gluteal projection after lower body lift. Aesthetic Surg J 37: 1012-1021.

14 Bayat A, Grouther DA, Ferguson MW (2003) Skin scarring. BMJ: British Medical Journal 326: 88-92.

15 Chugay N, Chugay P, Shiffman M (2004) Body sculpting with silicone implants. New York: Springer, USA. 